\title{
MACDONALD POLYNOMIALS IN SUPERSPACE: CONJECTURAL DEFINITION AND POSITIVITY CONJECTURES
}

\author{
O. BLONDEAU-FOURNIER, P. DESROSIERS, L. LAPOINTE, AND P. MATHIEU
}

\begin{abstract}
We introduce a conjectural construction for an extension to superspace of the Macdonald polynomials. The construction, which depends on certain orthogonality and triangularity relations, is tested for high degrees. We conjecture a simple form for the norm of the Macdonald polynomials in superspace, and a rather non-trivial expression for their evaluation. We study the limiting cases $q=0$ and $q=\infty$, which lead to two families of Hall-Littlewood polynomials in superspace. We also find that the Macdonald polynomials in superspace evaluated at $q=t=0$ or $q=t=\infty$ seem to generalize naturally the Schur functions. In particular, their expansion coefficients in the corresponding Hall-Littlewood bases appear to be polynomials in $t$ with nonnegative integer coefficients. More strikingly, we formulate a generalization of the Macdonald positivity conjecture to superspace: the expansion coefficients of the Macdonald superpolynomials expanded into a modified version of the Schur superpolynomial basis (the $q=t=0$ family) are polynomials in $q$ and $t$ with nonnegative integer coefficients.
\end{abstract}

\section{INTRODUCTION}

The Macdonald polynomials [15] can be defined by: (1) their orthogonality with respect to a certain scalar product, depending on two parameters $q, t$, and (2) their triangular decomposition in the monomial basis with respect to the dominance ordering. The aim of this work is to study the possible extension of the Macdonald polynomials to superspace along this precise constructive procedure, namely by enforcing triangularity and orthogonality. In the Jack polynomial limit $\left(q=t^{\alpha}\right.$ and $\left.t \rightarrow 1\right)$, we recall that such an extension to superspace was constructed in [3, 5]. It is important to note that while in the Jack's case the construction could rely on the supersymmetric version of the trigonometric Calogero-Moser-Sutherland model [20, 2], the supersymmetric version of the Macdonald operators and the Ruijsenaars-Schneider Hamiltonian are still elusive.

We introduce in Section 2 some preliminary results concerning the Macdonald polynomials and formulate the strategy to be followed in order to investigate their generalization without the support of an eigenvalue problem. The notion of superspace and the relevant properties of the corresponding extension of the Jack polynomials are briefly reviewed in Section 3. The stage is then prepared for the exploratory investigation of the Macdonald polynomials in superspace (also called Macdonald superpolynomials) presented in Section 4: a previously conjectured form of the scalar product [5, Conj. 34] is invalidated (no solution to the problem exist), but by introducing a slight deformation of this scalar product we end up with a new setup for the construction of the Macdonald polynomials in superspace. The correctness of this construction is substantiated here by the computation of many non-trivial examples which solve (for high degrees) the overdetermined systems of equations resulting from the imposed orthogonality and triangularity. Moreover, we present in the last subsections many conjectures that provide further evidence of

2000 Mathematics Subject Classification. 05E05 (Primary), 81Q60 and 33D52 (Secondary). 
the validity of the construction. In Section 4.3, we give conjectures for the norm, the integral version and the evaluation of the Macdonald superpolynomials. In Section 4.4 we study various limiting cases of the Macdonald superpolynomials. The limiting cases $q=0$ and $q=\infty$, which lead to two distinct families of Hall-Littlewood polynomials in superspace, are worth pointing out. We also find that the Macdonald polynomials in superspace evaluated at $q=t=0$ or $q=t=\infty$ seem to provide natural generalizations of the Schur functions. In particular, we conjecture in Section 4.5 that their expansion coefficients in the corresponding Hall-Littlewood bases are polynomials in $t$ with nonnegative integer coefficients. Section 4.5 culminates with the most striking result of the article: the extension of the Macdonald positivity conjecture to superspace. Concluding observations are made in Section 5, and finally, tables of Macdonald superpolynomials and Kostka coefficients are given at the end of the article.

\section{Macdonald POLynomials}

A partition $\lambda=\left(\lambda_{1}, \lambda_{2}, \ldots\right)$ of degree $d$ is a vector of non-negative integers such that $\lambda_{i} \geq \lambda_{i+1}$ for all $i$ and such that $\sum_{i} \lambda_{i}=d$. The Macdonald polynomials $P_{\lambda}=P_{\lambda}(x ; q, t)$, in the variables $x=x_{1}, x_{2}, \ldots, x_{N}$, are characterized by the two conditions

$$
\begin{aligned}
& \text { 1) } P_{\lambda}=m_{\lambda}+\text { lower terms, } \\
& \text { 2) }\left\langle\left\langle P_{\lambda} \mid P_{\mu}\right\rangle_{q, t}=0 \text { if } \lambda \neq \mu,\right.
\end{aligned}
$$

where $N$ is assumed to be larger than the degree of $\lambda$. The triangular decomposition is given with respect to the usual dominance order on partitions:

$$
\mu \leq \lambda \quad \text { iff } \quad|\mu|=|\lambda| \quad \text { and } \quad \mu_{1}+\cdots+\mu_{i} \leq \lambda_{1}+\cdots+\lambda_{i} \quad \forall i,
$$

where $|\lambda|$ stands for the degree of the partition. The monomial functions $m_{\lambda}$, in which the triangular expansion is formulated, are given by

$$
m_{\lambda}=\sum_{\sigma \in S_{N}}^{\prime} x_{\sigma(1)}^{\lambda_{1}} \cdots x_{\sigma(N)}^{\lambda_{N}},
$$

where the prime indicates a sum over distinct permutations. Note that we consider in the equation that $\lambda_{\ell+1}=\cdots=\lambda_{N}=0$ if the partition $\lambda$ is of length $\ell=\ell(\lambda)$ (the number of its non-zero parts). The orthogonality relation is defined in the power-sum basis

$$
p_{\lambda}=p_{\lambda_{1}} \cdots p_{\lambda_{\ell}} \quad \text { with } \quad p_{r}=\sum_{i=1}^{N} x_{i}^{r}
$$

by

where

$$
\left\langle\left\langle p_{\lambda} \mid p_{\mu}\right\rangle\right\rangle_{q, t}=z_{\lambda} \delta_{\lambda, \mu} \prod_{i=1}^{\ell(\lambda)} \frac{1-q^{\lambda_{i}}}{1-t^{\lambda_{i}}}
$$

$$
z_{\lambda}=\prod_{i \geq 1} i^{n_{\lambda}(i)} n_{\lambda}(i) !
$$

$n_{\lambda}(i)$ is the number of parts in $\lambda$ equal to $i$.

The Macdonald polynomials can be constructed, following characterization (2.1), using the Gram-Schmidt orthogonalization process (choosing any total ordering compatible with the dominance ordering). We should emphasize that imposing the triangularity with respect to the (partial) dominance order diminishes the number of unknowns in the Gram-Schmidt process 
and thus leads to over-determined systems of equations (see Section 2.1). Therefore, the question of existence needs to be settled separately. The standard procedure is to show that these polynomials are eigenfunctions of some operators, the Macdonald operators in the present case. The existence of non-trivial solutions to an overdetermined system is often the hallmark of an underlying integrable model. This is indeed the case here: the operators are actually related to the Ruijsenaars-Schneider Hamiltonian [19] (see also for instance [8, Sect. 1] and [18, Sect. 7.6.2]).

2.1. Exploring generalizations of the Macdonald polynomials: a simple strategy. The problem we consider here is to explore the existence of a particular extension of the Macdonald polynomials (to be formulated precisely below) in the absence of an underlying differential operator with respect to which the sought-after generalized polynomials would be eigenfunctions. The previous remark concerning the over-determinate nature of the corresponding system points toward a simple exploratory strategy that we now spell out plainly.

First, we stress that the consistency of the construction of families of polynomials characterized by orthogonality and triangularity is verified separately for each value of the degree of the partitions. It is clear that for low values of the degree, where the ordering is total, there will always exist a solution: there is an equal number of unknowns and equations. Stated differently (cf. [16, p. 9]), the Gram-Schmidt orthogonalization process ensures a unique solution to the conditions (1) in (2.1) and a weaker form of (2), namely:

$$
\left\langle\left\langle P_{\lambda} \mid P_{\mu}\right\rangle\right\rangle_{q, t}=0 \quad \text { if } \quad \lambda>\mu \text {. }
$$

However, for the first value $n$ of the degree at which not all partitions of $n$ are comparable in the dominance ordering, one encounters an over-determined system. Then, the critical test amounts to verify the orthogonality of the polynomials labeled by partitions that cannot be compared. The verification of these orthogonality relations provides a strong hint for the existence of these polynomials under construction. On the other hand, if it fails, this suffices to prove that the characterization by orthogonality and triangularity is not correct. Moreover, one expects this failure to show up for pairs of non-comparable partitions of lowest degree.

Let us illustrate this simple methodology for the ordinary Macdonald polynomials. The lowest degree at which the dominance ordering is not total is $n=6$. Indeed, two pairs of partitions cannot be compared: $(3,1,1,1)$ vs $(2,2,2)$ and $(3,3)$ vs $(4,1,1)$ (cf. Fig. 1(a)). Let us consider the construction of $P_{(3,1,1,1)}$ and $P_{(2,2,2)}$. Denote $(3,1,1,1)$ and $(2,2,2)$ by $\lambda^{(1)}$ and $\lambda^{(2)}$ respectively. Clearly, we have

$$
\lambda^{(i)} \geq(2,2,1,1) \geq(2,1,1,1,1) \geq(1,1,1,1,1,1), \quad i=1,2 .
$$

One thus constructs successively $P_{(1,1,1,1,1,1)}, P_{(2,1,1,1,1)}, P_{(2,2,1,1)}$, and then, separately, each $P_{\lambda^{(i)}}$, which are both of the form:

$$
P_{\lambda^{(i)}}=m_{\lambda^{(i)}}+a_{1}^{(i)} m_{(2,2,1,1)}+a_{2}^{(i)} m_{(2,1,1,1,1)}+a_{3}^{(i)} m_{(1,1,1,1,1,1)},
$$

with given coefficients $a_{j}^{(i)}$ (depending on $q$ and $t$ ) obtained by imposing:

$$
\left\langle\left\langle P_{\lambda^{(i)}} \mid P_{\mu}\right\rangle\right\rangle_{q, t}=0 \quad \text { for } \quad \mu \in\{(2,2,1,1),(2,1,1,1,1),(1,1,1,1,1,1)\} .
$$

The consistency test is to verify the orthogonality relation for the two polynomials labeled by non-comparable partitions, namely:

$$
\left\langle\left\langle P_{\lambda^{(1)}} \mid P_{\lambda^{(2)}}\right\rangle\right\rangle_{q, t}=0
$$


which is known to be satisfied in the present case.

FigURE 1. Hasse diagrams of the dominance ordering on partitions of degree 6 and on superpartitions of degree (4|1). The (super)partitions that can be compared are related by arrows pointing towards the lowest (super)partitions.

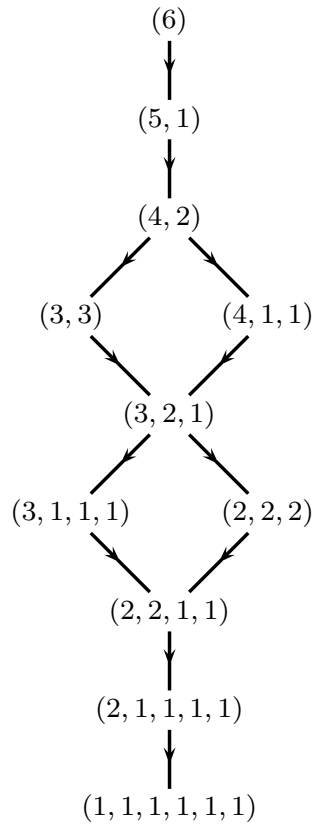

(a)

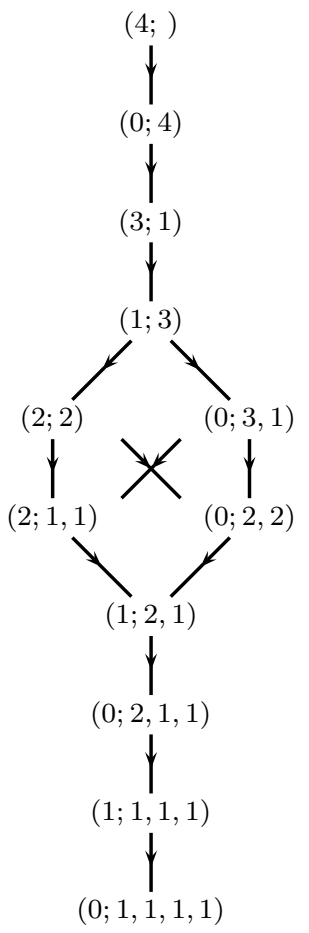

(b)

\section{Symmetric POlynomials in SUPERSPaCe}

Superspace refers to a pairing of each coordinate $x_{i}$ with an anticommuting partner $\theta_{i}$, where $\theta_{i}^{2}=0$. A polynomial in superspace (also called a superpolynomial) is a polynomial in the usual (commuting) $N$ variables $x_{1}, \cdots, x_{N}$ and the $N$ Grassmaniann variables $\theta_{1}, \cdots, \theta_{N}$. It is said to be symmetric if it remains invariant with respect to the simultaneous interchange of $x_{i} \leftrightarrow x_{j}$ and $\theta_{i} \leftrightarrow \theta_{j}$ for any $i, j[2]$. In other words, introducing for any $\sigma \in S_{N}$,

$$
\mathcal{K}_{\sigma}=\kappa_{\sigma} K_{\sigma}, \quad \text { where }\left\{\begin{aligned}
K_{\sigma}:\left(x_{1}, \ldots, x_{N}\right) & \mapsto\left(x_{\sigma(1)}, \ldots, x_{\sigma(N)}\right) \\
\kappa_{\sigma}:\left(\theta_{1}, \ldots, \theta_{N}\right) & \mapsto\left(\theta_{\sigma(1)}, \ldots, \theta_{\sigma(N)}\right),
\end{aligned}\right.
$$

then a polynomial in superspace $P(x ; \theta)$, with $x=\left(x_{1}, \ldots, x_{N}\right)$ and $\theta=\left(\theta_{1}, \ldots, \theta_{N}\right)$, is symmetric when

$$
\mathcal{K}_{\sigma} P(x ; \theta)=P(x ; \theta) \quad \text { for all } \sigma \in S_{N} .
$$

Symmetric superpolynomials are labeled by superpartitions [2]. A superpartition $\Lambda$ is a pair of partitions $\left(\Lambda^{a} ; \Lambda^{s}\right)=\left(\Lambda_{1}, \ldots, \Lambda_{m} ; \Lambda_{m+1}, \ldots, \Lambda_{N}\right)$, where $\Lambda^{a}$ is a partition with $m$ distinct parts (with possibly one of those parts equal to 0 ), and $\Lambda^{s}$ is a (regular) non-increasing partition 1$] \mathrm{A}$

\footnotetext{
${ }^{1}$ When $m=0, \Lambda=(; \lambda)$ can be thought as the ordinary partition $\lambda$ and the semi-column is omitted.
} 
superpartition $\Lambda$ of the above form is said to have degree $(n \mid m)$, written for short as $\operatorname{deg}(\Lambda)=$ $(n \mid m)$, when $\sum_{i} \Lambda_{i}=n$. We refer to $m$ and $n$ respectively as the fermionic degree and total degree of $\Lambda$. For example, there are seven superpartitions of degree (3|1):

$$
(0 ; 1,1,1),(0 ; 2,1),(0 ; 3),(1 ; 1,1),(1 ; 2),(2 ; 1),(3 ;),
$$

and twelve at degree (4|1) (listed in Fig. 1(b)).

To every superpartition $\Lambda$ we can associate a unique partition $\Lambda^{*}$ obtained by deleting the semicolon and reordering the parts in non-increasing order. A diagrammatic representation of $\Lambda$ is given by the Ferrers diagram of $\Lambda^{*}$ with circles added at the end of every part of $\Lambda^{a}$, and ordered in length as if a circle was a half-box. Finally, we denote by $\Lambda^{\circledast}$ the partition whose Ferrers diagram is obtained from that of $\Lambda$ by replacing circles by boxes. For instance, we have:
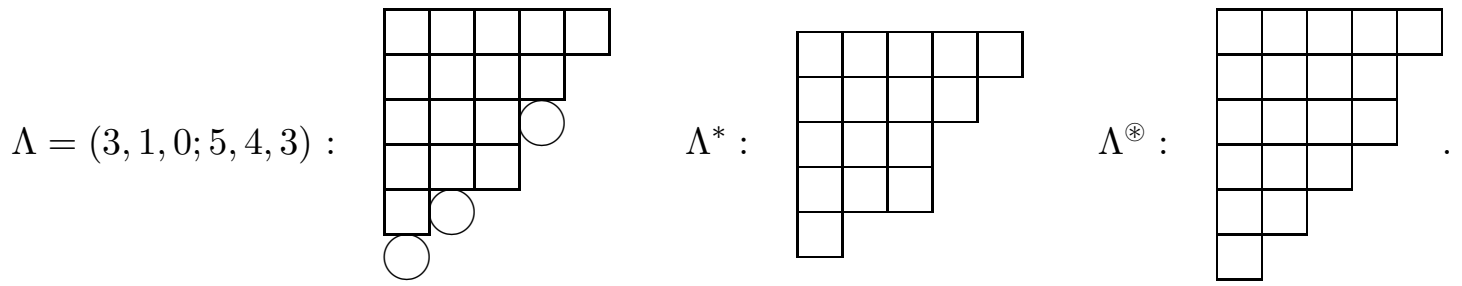

Notice that both $\Lambda^{*}$ and $\Lambda^{\circledast}$ are ordinary partitions, of respective degree $n$ and $n+m$, if $\operatorname{deg}(\Lambda)=(n \mid m)$.

The natural extension of the usual dominance ordering to superpartitions is the following [6]:

$$
\Omega \leq \Lambda \quad \text { iff } \quad \operatorname{deg}(\Lambda)=\operatorname{deg}(\Omega), \quad \Omega^{*} \leq \Lambda^{*} \quad \text { and } \quad \Omega^{\circledast} \leq \Lambda^{\circledast} .
$$

We stress that two pairs of ordinary partitions, $\Omega^{*}$ vs $\Lambda^{*}$ and $\Omega^{\circledast}$ vs $\Lambda^{\circledast}$, are compared with respect to the usual dominance ordering (2.2). The lowest degree at which this ordering is not total is (4|1) (see Fig. 1(b)). In that case, two pairs of superpartitions cannot be compared: $(2 ; 1,1)$ vs $(0 ; 2,2)$ and $(2 ; 2)$ vs $(0 ; 3,1)$. For instance, for the first pair, we have:

$$
\begin{array}{lll}
\Lambda=(2 ; 1,1), & \Lambda^{*}=(2,1,1), & \Lambda^{\circledast}=(3,1,1), \\
\Omega=(0 ; 2,2), & \Omega^{*}=(2,2,0), & \Omega^{\circledast}=(2,2,1),
\end{array}
$$

so that

$$
\Lambda^{*}<\Omega^{*} \quad \text { but } \quad \Lambda^{\circledast}>\Omega^{\circledast} .
$$

We now introduce the two relevant classical bases of symmetric polynomials in superspace [4]. The first one is the extension of the monomial polynomials defined as

$$
m_{\Lambda}(x ; \theta)=\sum_{\sigma \in S_{N}}{ }^{\prime} \mathcal{K}_{\sigma}\left(\theta_{1} \cdots \theta_{m} x_{1}^{\Lambda_{1}} \cdots x_{N}^{\Lambda_{N}}\right),
$$

where the sum is over distinct permutations. The other one is the generalization of the powersum symmetric functions

$$
p_{\Lambda}=\tilde{p}_{\Lambda_{1}} \cdots \tilde{p}_{\Lambda_{m}} p_{\Lambda_{m+1}} \cdots p_{\Lambda_{\ell}}, \quad \text { where } \quad \tilde{p}_{k}=\sum_{i} \theta_{i} x_{i}^{k} \quad \text { and } \quad p_{\ell}=\sum_{i} x_{i}^{\ell},
$$

where $k \geq 0$ and $\ell \geq 1$.

We conclude this review section by recalling the definition of the precise objects we want to deform, the Jack superpolynomials. We denote them as $P_{\Lambda}=P_{\Lambda}^{(\alpha)}(x, \theta)$; they are characterized 
by the two conditions [5]:

$$
\begin{aligned}
& \text { 1) } P_{\Lambda}=m_{\Lambda}+\text { lower terms, } \\
& \text { 2) }\left\langle\left\langle P_{\Lambda} \mid P_{\Omega}\right\rangle_{\alpha}=0 \text { if } \Lambda \neq \Omega,\right.
\end{aligned}
$$

where the scalar product is defined as

$$
\left\langle\left\langle p_{\Lambda} \mid p_{\Omega}\right\rangle\right\rangle_{\alpha}=(-1)^{\left(\begin{array}{c}
m \\
2
\end{array}\right)} \alpha^{\ell(\Lambda)} z_{\Lambda^{s}} \delta_{\Lambda, \Omega}
$$

with $z_{\Lambda^{s}}$ defined in (2.6) and $\ell(\Lambda)=m+\ell\left(\Lambda^{s}\right)=\ell\left(\Lambda^{\circledast}\right)$. The existence of such a basis is proved in [5] using the supersymmetric extension of the Calogero-Moser-Sutherland model.

\section{The QUeSt For Macdonald POlynomials in SUPerspace}

We are now in position to explore the existence of the superspace extension of the Macdonald polynomials obtained by a suitable generalization of the construction reviewed previously for the limiting cases of the ordinary Macdonald polynomials and the Jack superpolynomials.

4.1. Invalidation of the original conjecture. We first consider the conjecture in [5, Conj. 34] formulated in terms of the conditions (3.10) and the dominance ordering (3.5), but with the scalar product (3.11) replaced by the following natural $(q, t)$ deformation 2

$$
\left\langle\left\langle p_{\Lambda} \mid p_{\Omega}\right\rangle\right\rangle_{q, t}^{(F)}=(-1)^{\left(\begin{array}{c}
m \\
2
\end{array}\right)} z_{\Lambda}(q, t) \delta_{\Lambda, \Omega}
$$

where

$$
\tilde{z}_{\Lambda}(q, t)=z_{\Lambda^{s}} \prod_{i=1}^{\ell(\Lambda)} \frac{1-q^{\Lambda_{i}^{\circledast}}}{1-t^{\Lambda_{i}^{\circledast}}} .
$$

This reduces to (3.11) when $q=t^{\alpha}$ and $t \rightarrow 1$. The rationale motivating this precise form of the conjectured scalar product is rooted in the naturality of the generalized form of the reproducing kernel:

$$
\prod_{i, j} \frac{\left(t x_{i} y_{j}+t \theta_{i} \phi_{j} ; q\right)_{\infty}}{\left(x_{i} y_{j}+\theta_{i} \phi_{j} ; q\right)_{\infty}}=\sum_{\Lambda}(-1)^{\left(\begin{array}{c}
m \\
2
\end{array}\right)} \tilde{z}_{\Lambda}(q, t)^{-1} p_{\Lambda}(x, \theta) p_{\Lambda}(y, \phi),
$$

where $(a ; q)_{\infty}=\prod_{n>0}\left(1-a q^{n}\right)$ (for the details of this correspondence, in particular, the way $\tilde{z}_{\Lambda}(q, t)$ is read off this relation, we refer to a similar analysis in [4, Theo. 33]). Indeed, for all the cases studied previously, namely the classical symmetric functions [4] and the Jack polynomials [5], the superspace extension of the kernel is obtained by simply replacing $x_{i} y_{j}$ by $x_{i} y_{j}+\theta_{i} \phi_{j}$.

As explained in Section 2.1, displaying a single counter-example suffices to invalidate the conjecture. And the simplest such counter-example is expected to arise at the lowest degree $(n \mid m)$ where the ordering is not total, which is $(4 \mid 1)$. This is indeed so: the scalar product of $P_{(2 ; 1,1)}$ and $P_{(0 ; 2,2)}$, polynomials whose labeling superpartitions are not comparable (cf. (3.6)(3.7)), is found to be non-zero 3

2 The conjecture was originally presented with respect to the ordering [4, Corr. 7]:

$$
\Omega \leq^{\prime} \Lambda \quad \text { iff } \quad \operatorname{deg}(\Lambda)=\operatorname{deg}(\Omega) \quad \text { and } \quad\left\{\begin{array}{l}
\Omega^{*}<\Lambda^{*} \quad \text { or } \\
\Omega^{*}=\Lambda^{*} \quad \text { and } \quad \Omega^{\circledast} \leq \Lambda^{\circledast} .
\end{array}\right.
$$

The ordering $\leq^{\prime}$ leads to less over-determined systems of equations in our construction than the ordering $\leq$ given that more pairs are comparable in the ordering $\leq^{\prime}$ than in the ordering $\leq$. Nevertheless we have verified that the conjecture also fails using the ordering $\leq^{\prime}$ (this time at degree $(6 \mid 1)$ ).

${ }^{3}$ It vanishes only in the Jack superpolynomial limit $q=t^{\alpha}$ and $t \rightarrow 1$ and for $q=t$. 
4.2. A new conjecture. Although (4.1) is natural, the correctness of the Jack-limiting behavior is not fully constraining. Actually, there is a whole family of acceptable deformations of (3.11). In particular, the form (3.11) depends rather weakly upon the parts in $\Lambda^{a}$ : it only depends upon their number $m$ via the factor $(-1)^{\left(\begin{array}{c}m \\ 2\end{array}\right)} \alpha^{m}$. This suggests to investigate the following simple deformation of $\tilde{z}_{\Lambda}$ which amounts to replace the portion of the product depending upon $\Lambda^{a}$ by

$$
\prod_{i=1}^{m} \frac{1-q^{\Lambda_{i}+1}}{1-t^{\Lambda_{i}+1}} \rightarrow \prod_{i=1}^{m} \frac{1-q^{\Lambda_{i}+1}}{1-\tau^{\Lambda_{i}+1}}
$$

where $\tau$ is a parameter depending on $q$ and $t$ that needs to be determined. This form can be obtained from the generalized kernel :

$$
K^{(\tau)}(x, \theta ; y, \phi)=\prod_{i, j} \frac{\left(\tau x_{i} y_{j}+\tau \theta_{i} \phi_{j} ; q\right)_{\infty}\left(t x_{i} y_{j} ; q\right)_{\infty}}{\left(x_{i} y_{j}+\theta_{i} \phi_{j} ; q\right)_{\infty}\left(\tau x_{i} y_{j} ; q\right)_{\infty}}
$$

which reduces to (4.3) when $\tau=t$. Call the corresponding $\tau$-dependent scalar product $\left\langle\langle\mid\rangle_{q, t}^{(\tau)}\right.$. To fix $\tau$, we again consider the lowest degree where the dominance ordering is not total, namely $(4 \mid 1)$. As already stressed, at this degree two pairs of superpartitions are non-comparable: $(2 ; 1,1)$ vs $(0 ; 2,2)$ and $(2 ; 2)$ vs $(0 ; 3,1)$. By enforcing the orthogonality of $P_{(2 ; 1,1)}$ and $P_{(0 ; 2,2)}$, one gets:

$$
\left\langle\left\langle P_{(2 ; 1,1)} \mid P_{(0 ; 2,2)}\right\rangle\right\rangle_{q, t}^{(\tau)}=0 \quad \Longleftrightarrow \quad \tau=q^{-1}
$$

As a non-trivial compatibility check, we verify that $P_{(2 ; 2)}$ and $P_{(0 ; 3,1)}$ are also orthogonal for this choice of $\tau$.

With $\tau=q^{-1}$, the kernel (4.5) simplifies drastically as an infinite number of factors cancel. It reduces to:

$$
K^{(1 / q)}(x, \theta ; y, \phi)=\prod_{i, j}\left(1-\frac{\theta_{i} \phi_{j}}{q-x_{i} y_{j}}\right) \frac{\left(t x_{i} y_{j} ; q\right)_{\infty}}{\left(x_{i} y_{j} ; q\right)_{\infty}}
$$

The combination $q-x_{i} y_{j}$ in (4.6) is not natural: $q$ usually appears as a scaling factor of the variables. This can easily be cured by rescaling the anticommuting variable $\theta_{i}$ by a factor $-q$. This modification does not affect the resulting Macdonald polynomials since all their components are multiplied by the same number of anticommuting variables (this alteration only changes their norm). The effect of the replacement $\theta_{i} \rightarrow-q \theta_{i}$ in the kernel is to transform it into

$$
K(x, \theta ; y, \phi)=\prod_{i, j}\left(1+\frac{\theta_{i} \phi_{j}}{1-q^{-1} x_{i} y_{j}}\right) \frac{\left(t x_{i} y_{j} ; q\right)_{\infty}}{\left(x_{i} y_{j} ; q\right)_{\infty}}=K_{0}(x ; y) \prod_{i, j}\left(1+\frac{\theta_{i} \phi_{j}}{1-q^{-1} x_{i} y_{j}}\right) .
$$

where $K_{0}(x ; y)$ is the kernel in the absence of anticommuting variables. (The last expression is to be compared with [5, eq. (5.14)] in the Jack case.) With

$$
K(x, \theta ; y, \phi)=\sum_{\Lambda}(-1)^{\left(\begin{array}{c}
m \\
2
\end{array}\right)} z_{\Lambda}(q, t)^{-1} p_{\Lambda}(x, \theta) p_{\Lambda}(y, \phi),
$$

our new scalar product reads:

$$
\left\langle\left\langle p_{\Lambda} \mid p_{\Omega}\right\rangle\right\rangle_{q, t}=(-1)^{\left(\begin{array}{c}
m \\
2
\end{array}\right)} z_{\Lambda}(q, t) \delta_{\Lambda, \Omega},
$$

where

$$
z_{\Lambda}(q, t)=z_{\Lambda^{s}} q^{\left|\Lambda^{a}\right|} \prod_{i=1}^{\ell\left(\Lambda^{s}\right)} \frac{1-q^{\Lambda_{i}^{s}}}{1-t^{\Lambda_{i}^{s}}}
$$


indeed, a rather simple form. We note however that this does not precisely reduces to the expression $\left\langle\left\langle p_{\Lambda} \mid p_{\Omega}\right\rangle\right\rangle_{\alpha}$ written in (3.11), but rather to $\alpha^{-m}\left\langle\left\langle p_{\Lambda} \mid p_{\Omega}\right\rangle\right\rangle_{\alpha}$. However, such a modification does not affect the resulting Jack superpolynomials since only polynomials with the same fermionic degree $m$ are compared.

It is thus natural to conjecture the existence of a superspace generalization of the Macdonald polynomials, written $P_{\Lambda}=P_{\Lambda}(x, \theta ; q, t)$, as follows:

Conjecture 1. For each superpartition $\Lambda$ there is a unique symmetric superpolynomial $P_{\Lambda}$ such that:

$$
\begin{aligned}
& \text { 1) } P_{\Lambda}=m_{\Lambda}+\text { lower terms, } \\
& \text { 2) }\left\langle\left\langle P_{\Lambda} \mid P_{\Omega}\right\rangle_{q, t}=0 \quad \text { if } \Lambda \neq \Omega .\right.
\end{aligned}
$$

where lower terms are considered with respect to the ordering (3.5) and the scalar product is defined in (4.9) and (4.10).

This conjecture has been heavily tested. The cases that have been checked are listed in Table 1. Examples of Macdonald polynomials in superspace are presented in Table 2.

TABLE 1. The different degrees that have been tested at which the system is over-determined.

\begin{tabular}{|l|c|c|c|c|c|c|c|c|c|}
\hline \hline degree $(n \mid m)$ & $(4 \mid 1)$ & $(5 \mid 2)$ & $(5 \mid 1)$ & $(6 \mid 3)$ & $(6 \mid 2)$ & $(6 \mid 1)$ & $(7 \mid 3)$ & $(8 \mid 3)$ & $(9 \mid 4)$ \\
\# equations & 66 & 136 & 171 & 45 & 378 & 435 & 171 & 528 & 45 \\
\# unknowns & 64 & 128 & 163 & 43 & 351 & 401 & 163 & 482 & 43 \\
\hline \hline
\end{tabular}

From the examples in Table 2, we observe that a property of the Macdonald polynomials that does not hold in superspace is the invariance with respect to $(q, t) \mapsto\left(q^{-1}, t^{-1}\right)$ : in general, whenever $m>0$ we have that

$$
P_{\Lambda}(x, \theta ; q, t) \neq P_{\Lambda}\left(x, \theta ; q^{-1}, t^{-1}\right)
$$

(cf. [15] property VI (4.14)-(iv) for $m=0$ ). This is already manifest from the simplest displayed case $P_{(1 ; 0)}$. In fact, if we set $\bar{P}_{\Lambda}(x, \theta ; q, t)=P_{\Lambda}\left(x, \theta ; q^{-1}, t^{-1}\right)$, then Conjecture 1 is equivalent to saying that $\bar{P}_{\Lambda}(x, \theta ; q, t)$ is the unique polynomial satisfying 1$)$ and 2$)$, but with the scalar product replaced by

$$
\left\langle\left\langle p_{\Lambda} \mid p_{\Omega}\right\rangle\right\rangle_{q, t}^{\prime}=(-1)^{\left(\begin{array}{c}
m \\
2
\end{array}\right)} \bar{z}_{\Lambda}(q, t) \delta_{\Lambda, \Omega}, \quad \text { where } \quad \bar{z}_{\Lambda}(q, t)=z_{\Lambda^{s}} t^{-\left|\Lambda^{a}\right|} \prod_{i=1}^{\ell\left(\Lambda^{s}\right)} \frac{1-q^{\Lambda_{i}^{s}}}{1-t^{\Lambda_{i}^{s}}}
$$

As will be explained below, this new scalar product is more convenient in certain circumstances since it allows to take the limit $q \rightarrow 0$.

4.3. The norm, the integral version and the specialization. For a box $s=(i, j) \in \Lambda$ (i.e., in row $i$ and column $j$ ), introduce the following arm-lengths and leg-lengths:

$$
\begin{array}{cc}
a(s)=\Lambda_{i}^{*}-j, & \tilde{a}(s)=\Lambda_{i}^{\circledast}-j, \\
l(s)=\left(\Lambda^{*}\right)_{j}^{\prime}-i, & \tilde{l}(s)=\left(\Lambda^{\circledast}\right)_{j}^{\prime}-i,
\end{array}
$$


where $\left(\Lambda^{*}\right)^{\prime}$ and $\left(\Lambda^{\circledast}\right)^{\prime}$ stand for the conjugate of the partitions $\Lambda^{*}$ and $\Lambda^{\circledast}$ respectively. We now define

$$
w_{\Lambda}(q, t)=\prod_{s \in \mathcal{B}(\Lambda)}\left(1-q^{a(s)+1} t^{\tilde{l}(s)}\right),
$$

where $\mathcal{B}(\Lambda)$ denotes the set of boxes in the diagram of $\Lambda$ that do not appear at the same time in a row containing a circle and in a column containing a circle (this excludes for instance the boxes $(3,1),(3,2)$ and $(5,1)$ of $\Lambda$ whose diagram is found in (3.4) $)$. These are the required ingredients for formulating our conjecture for the norm expression.

Conjecture 2. Let $P_{\Lambda}$ be the superpolynomial of Conjecture 1 and its norm be defined by

$$
\left\|P_{\Lambda}\right\|^{2}=(-1)^{\left(\begin{array}{c}
m \\
2
\end{array}\right)}\left\langle\left\langle P_{\Lambda} \mid P_{\Lambda}\right\rangle\right\rangle_{q, t} .
$$

Then, the norm is given by

$$
\left\|P_{\Lambda}\right\|^{2}=q^{\mid \Lambda^{a}} \mid \frac{w_{\Lambda}(q, t)}{w_{\Lambda^{\prime}}(t, q)}=q^{\left|\Lambda^{a}\right|} \prod_{s \in \mathcal{B}(\Lambda)} \frac{1-q^{a(s)+1} t^{\tilde{l}(s)}}{1-q^{\tilde{a}(s)} t^{l(s)+1}},
$$

where $\Lambda^{\prime}$ is the conjugate of $\Lambda$ (the superpartition whose diagram is obtained by interchanging the rows and the columns in the diagram of $\Lambda$ ).

This expression for the combinatorial norm generalizes naturally both that of the Macdonald polynomials [15, Eq. (VI.6.19)] and that of the Jack superpolynomials established in [13, 6]. For example, the above formula yields

$$
\left\|P_{(3,0 ; 1)}\right\|^{2}=\frac{q^{3}(1-q)^{2}(1+q)}{(1-t)\left(1-q^{2} t\right)} \quad \text { and } \quad\left\|P_{(2,1,0 ;)}\right\|^{2}=q^{3} .
$$

Given our conjectured norm expression, it is natural to guess the form of the proper integral version of the Macdonald superpolynomials:

Conjecture 3. The monomial expansion coefficients of the following normalization of the Macdonald superpolynomials

$$
J_{\Lambda}=w_{\Lambda^{\prime}}(t, q) P_{\Lambda}
$$

are polynomial in $q, t$ with integral coefficients (albeit not necessarily positive).

This is a natural generalization of the integral form of the usual Macdonald polynomials [15. Eq. (VI.8.3)] and the $(q, t)$-version of [5, Conj. 33]. The extension to superspace of the Macdonald positivity conjecture (see Conjecture 8) will be given in terms of $J_{\Lambda}$.

Finally, we turn to the conjectured expression for the specialization. Let $F(x ; \theta)$ be a polynomial in superspace of fermionic degree $m$ and suppose that $N \geq m$. The evaluation of $F(x ; \theta)$ is defined as

$$
\mathrm{E}_{N, m}[F(x ; \theta)]:=\left[\frac{\partial_{\theta_{m}} \cdots \partial_{\theta_{1}} F(x ; \theta)}{V_{m}(x)}\right]_{x_{1}=u_{1}, \cdots, x_{N}=u_{N}},
$$

where the specialization values $u_{i}$ are given by

$$
u_{i}=\frac{t^{i-1}}{q^{\max (m-i, 0)}}
$$

and where

$$
V_{m}(x)=\prod_{1 \leq i<j \leq m}\left(x_{i}-x_{j}\right)
$$


is the Vandermonde determinant in the variables $x_{1}, \ldots, x_{m}$.

The following conjecture involves a combinatorial number $\zeta_{\Lambda}$ that has no analogue for ordinary partitions. We consider the partial filling of the squares of $\Lambda$ defined as follows: in each fermionic square of $\Lambda$ write the number of bosonic squares above it; $\zeta_{\Lambda}$ is obtained by adding up these numbers. Here are three examples for which it is non-vanishing:

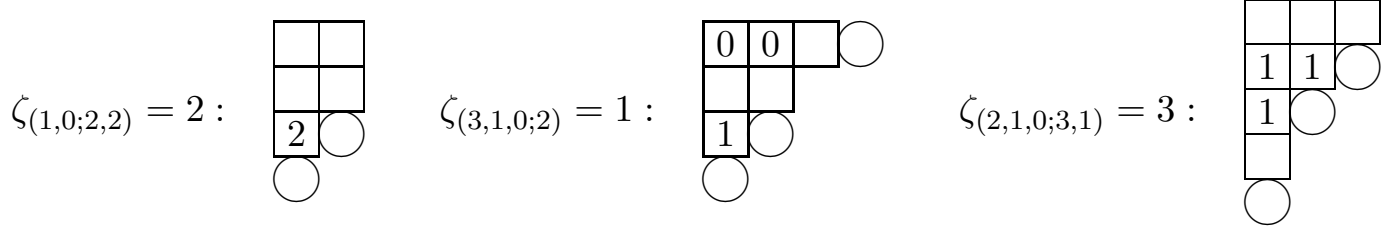

We also need to introduce, for $\Lambda$ a superpartition of fermionic degree $m$, the skew diagram $\mathcal{S} \Lambda=\Lambda^{\circledast} / \delta^{(m+1)}$, where $\delta^{(m)}$ is the staircase partition $(m-1, m-2, \ldots, 1,0)$, and the quantity $b(\lambda)=\sum_{i}(i-1) \lambda_{i}$, where $\lambda$ is a partition. Note that $b(\lambda / \mu)$ will stand for $b(\lambda)-b(\mu)$.

Conjecture 4. Let $\Lambda$ be of fermionic degree $m$ and suppose that $N \geq \ell\left(\Lambda^{\circledast}\right)$. Then the evaluation formula for the Macdonald superpolynomials $J_{\Lambda}$ defined in Conjecture 3 is:

$$
\mathrm{E}_{N, m}\left[J_{\Lambda}(x, \theta ; q, t)\right]=\frac{t^{\zeta_{\Lambda}} t^{b(\mathcal{S} \Lambda)}}{q^{(m-1)\left|\Lambda^{a} / \delta^{(m)}\right|-b\left(\Lambda^{a} / \delta^{(m)}\right)}} \prod_{(i, j) \in \mathcal{S} \Lambda}\left(1-q^{(j-1)} t^{N-(i-1)}\right)
$$

For $m=0$, this reduces to the usual evaluation formula for the Macdonald polynomials [15. Eq. (VI.6.11')], while for $q=t^{\alpha}$ and $t \rightarrow 1$, it reduces to that of the Jack superpolynomials [6, Theo. 26].

To illustrate this formula, consider the evaluation of $J_{(3,1,0 ; 1,1)}$. Here $m=3$ and we set $N=7$, so that $\left(x_{1}, \cdots, x_{7}\right)=\left(q^{-2}, t q^{-1}, t^{2}, t^{3}, t^{4}, t^{5}, t^{6}\right)$. We have

$$
(m-1)\left|\Lambda^{a} / \delta^{(m)}\right|-b\left(\Lambda^{a} / \delta^{(m)}\right)=2 \cdot 1-0=2, \quad b(\mathcal{S} \Lambda)=b(42111 / 321)=11-4=7 .
$$

In the following product the contributing boxes are $(1,4),(4,1),(5,1)$ :

$$
\prod_{(i, j) \in \mathcal{S}(\Lambda)}\left(1-q^{(j-1)} t^{N-(i-1)}\right)=\left(1-q^{3} t^{7}\right)\left(1-t^{4}\right)\left(1-t^{3}\right) .
$$

Finally, since $\zeta_{(3,1,0 ; 1,1)}=0$, we have

$$
\mathrm{E}_{7,3}\left[J_{(3,1,0 ; 1,1)}\right]=q^{-2} t^{7}\left(1-t^{3}\right)\left(1-t^{4}\right)\left(1-q^{3} t^{7}\right)
$$

4.4. Limiting cases. We recall that for any superpartition $\Lambda=(; \lambda)$ of fermionic degree $m=0$, the superpolynomial $P_{\Lambda}(x, \theta ; q, t)$ reduces to the standard Macdonald polynomial $P_{\lambda}(x ; q, t)$, whose distinct limiting cases [15] are summarized in Fig. 2. We now aim at generalizing this picture to superspace.

The simplest cases are those directly related to the Jack polynomials. Indeed, as previously mentioned, with $q=t^{\alpha}$ and $t \rightarrow 1$ in $P_{\Lambda}(x, \theta ; q, t)$, the monic Jack superpolynomial $P_{\Lambda}^{(\alpha)}(x, \theta)$ is recovered. Moreover, it has been previously established [5] that $P_{\Lambda}^{(\alpha)}(x, \theta)$ tends to $m_{\Lambda}(x, \theta)$ as $\alpha \rightarrow \infty$ while it tends to $(-1)^{\left(\begin{array}{c}m \\ 2\end{array}\right)} e_{\Lambda^{\prime}}(x, \theta)$ as $\alpha \rightarrow 0$, where the elementary superpolynomials are defined as

$$
e_{\Lambda}(x, \theta)=\tilde{e}_{\Lambda_{1}^{a}}(x, \theta) \cdots \tilde{e}_{\Lambda_{m}^{a}}(x, \theta) e_{\Lambda_{1}^{s}}(x) \cdots e_{\Lambda_{\ell}^{s}}(x),
$$


FIGURE 2. Classical limiting cases. The dotted arrows indicate limits not related to the Schur functions

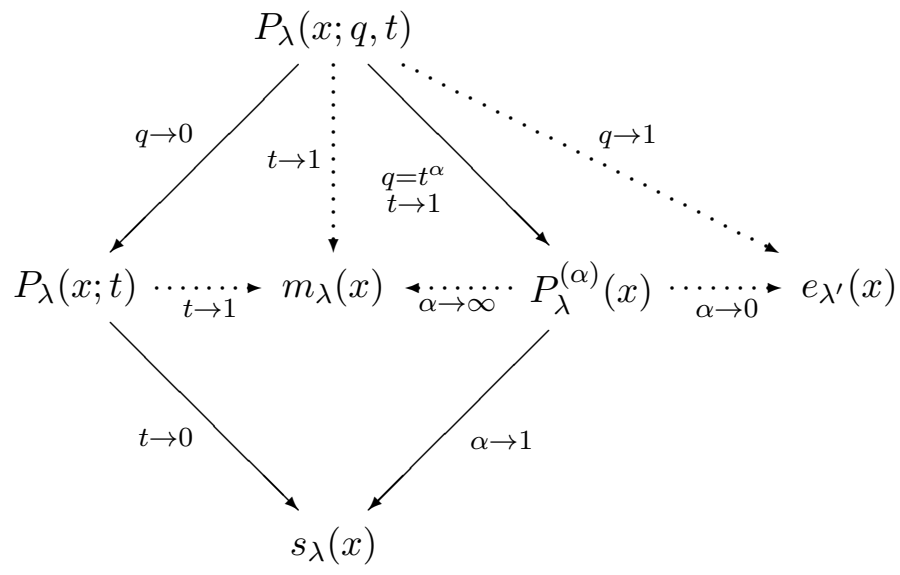

with

$$
\tilde{e}_{n}(x, \theta)=m_{\left(0 ; 1^{n}\right)}(x, \theta) \quad \text { and } \quad e_{n}(x)=m_{\left(1^{n}\right)}(x) .
$$

Similarly, for all tested Macdonald superpolynomials, we found

$$
P_{\Lambda}(x, \theta ; q, 1)=m_{\Lambda}(x, \theta) \quad \text { and } \quad P_{\Lambda}(x, \theta ; 1, t)=(-1)^{\left(\begin{array}{c}
m \\
2
\end{array}\right)} e_{\Lambda^{\prime}}(x, \theta) .
$$

This means that each dotted limit of Fig. 2 has a simple generalization to superspace.

Consider now the Hall-Littlewood limit. In the standard case, $P_{\lambda}(x ; t)$ is equal to $P_{\Lambda}(x ; q, t)$ whenever $q=0$, so it is natural to define the Hall-Littlewood superpolynomial as

$$
P_{\Lambda}(x, \theta ; t)=P_{\Lambda}(x, \theta ; 0, t) .
$$

From Table 2, we see that many superpolynomials $P_{\Lambda}$ simply reduce to $m_{\Lambda}$ when $q=0$. This extreme simplicity is, however, an artifact of the smallness of the degree of these examples. Another noteworthy feature is that the scalar product (4.9) is degenerate when $q=0$. Consequently, our construction based on the extension of the reproducing kernel does not provide a natural scalar product for the $P_{\Lambda}(x, \theta ; t)$ 's. In particular, their existence would not follow from a proof of the existence of the Macdonald polynomials in superspace.

There is however another possible generalization of the Hall-Littlewood polynomial to superspace. Indeed, the standard Macdonald polynomials are invariant under the transformation $(q, t) \mapsto\left(q^{-1}, t^{-1}\right)$, which implies that $P_{\lambda}\left(x ; t^{-1}\right)=P_{\lambda}(x ; \infty, t)$. This suggests to define $\bar{P}_{\Lambda}\left(x, \theta ; t^{-1}\right)=P_{\Lambda}(x, \theta ; \infty, t)$, which is equivalent to defining

$$
\bar{P}_{\Lambda}(x, \theta ; t)=\bar{P}_{\Lambda}(x, \theta ; 0, t),
$$

where $\bar{P}_{\Lambda}(x, \theta ; q, t)=P_{\Lambda}\left(x, \theta ; q^{-1}, t^{-1}\right)$. As could be expected from the non-symmetry in (4.12), one gets (see Table 2) that the families $\bar{P}_{\Lambda}(x, \theta ; t)$ and $P_{\Lambda}(x, \theta ; t)$ are distinct. The advantage of the family $\bar{P}_{\Lambda}$ is that it inherits a scalar product from (4.13).

Conjecture 5. The Hall-Littlewood superpolynomial $\bar{P}_{\Lambda}(x, \theta ; t)=P_{\Lambda}\left(x, \theta ; \infty, t^{-1}\right)$ is the unique superpolynomial satisfying 1) and 2) of Conjecture 1 but with the second condition replaced by

$$
\left\langle\left\langle\bar{P}_{\Omega}, \bar{P}_{\Lambda}\right\rangle\right\rangle_{0, t}^{\prime}=0, \quad \Omega \neq \Lambda \text {. }
$$

where the scalar product is that given in (4.13) when $q=0$. 
It is tempting to conclude from the above conjecture that the appropriate Hall-Littlewood superpolynomials should be the $\bar{P}_{\Lambda}$, but as we will see shortly, both families enjoy remarkable and somewhat entangled properties. A similar separation phenomenon occurs for the Schur superpolynomials. We know from Fig. 2 that the standard Schur polynomial $s_{\lambda}$ can be seen either as the Hall-Littlewood polynomial $P_{\lambda}(x ; 0)$ or as the Jack polynomial $P_{\lambda}^{(1)}(x)$. The generalization of the limiting cases to superspace is given in Fig. 3 and provides us with three possible Schur superpolynomials that do not depend upon any extra parameter:

$$
s_{\Lambda}(x, \theta)=P_{\Lambda}(x, \theta ; 0,0), \quad s_{\Lambda}^{\mathrm{Jack}}(x, \theta)=P_{\Lambda}(x, \theta ; 1,1), \quad \bar{s}_{\Lambda}(x, \theta)=P_{\Lambda}(x, \theta ; \infty, \infty),
$$

These superpolynomials are respectively equal to the limits $t \rightarrow 0, t \rightarrow 1, t \rightarrow \infty$ of

$$
s_{\Lambda}(x, \theta ; t)=P_{\Lambda}(x, \theta ; t, t) .
$$

We stress that when $\Lambda=(; \lambda)$ has fermionic degree $m=0$, the latter reduces to $P_{\lambda}(x ; t, t)$, which is independent of $t$ and equal to the standard Schur polynomial $s_{\lambda}(x)$. According to Conjecture 1, the one-parameter Schur polynomials $s_{\Lambda}(x, \theta ; t)$ should be the unique triangular monic superpolynomials that are also orthogonal with respect to the following scalar product:

$$
\left\langle\left\langle p_{\Lambda} \mid p_{\Omega}\right\rangle\right\rangle_{t, t}=\left.(-1)^{\left(\begin{array}{c}
m \\
2
\end{array}\right)} z_{\Lambda^{s}}\right|^{\left|\Lambda^{a}\right|} \delta_{\Lambda, \Omega} .
$$

Note that the latter equation is well defined for all $t \neq 0, \infty$. From a combinatorial point of view however, the positivity properties described below confirm that the cases $t=0, \infty$ are the most interesting even though the scalar product is degenerate in those cases.

FIGURE 3. Limiting cases in superspace

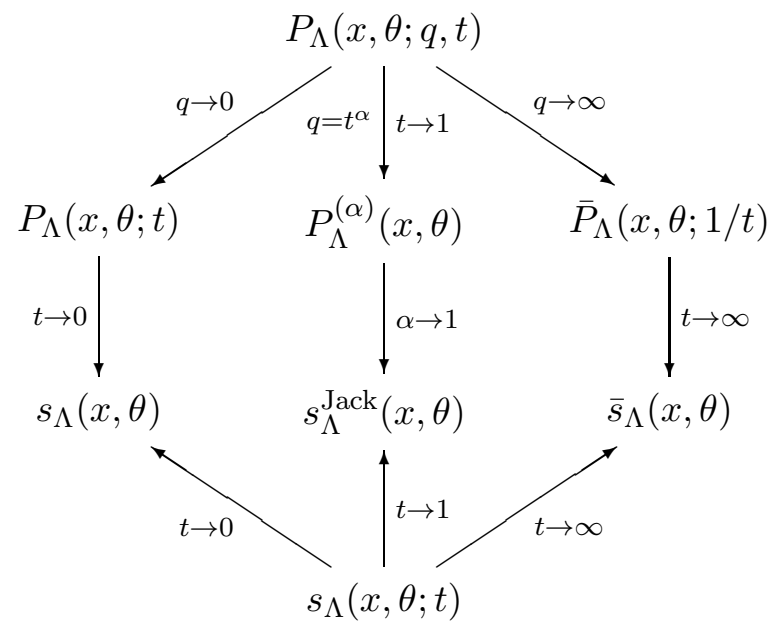

4.5. A Macdonald positivity conjecture in superspace. As was just mentioned, by setting $\alpha=1$ in the Jack superpolynomial case, one obtains a candidate superspace version of the Schur polynomials 4 However, these superpolynomials do not seem to have distinguished combinatorial

\footnotetext{
${ }^{4}$ We stress that these are completely different from the so-called supersymmetric Schur polynomials (see e.g., 21]) which have no relation with anticommuting variables. For more details, see [4, Remark 14].
} 
properties such as positivity and integrality. This is neatly illustrated by the following example:

$$
\begin{aligned}
s_{(1,0 ; 3)}^{\mathrm{Jack}}= & \lim _{t \rightarrow 1} P_{(1,0 ; 3)}(x, \theta ; t, t) \\
& =m_{(1,0 ; 3)}+\frac{1}{2} m_{(2,0 ; 2)}+\frac{7}{8} m_{(1,0 ; 2,1)}+\frac{1}{4} m_{(2,0 ; 1,1)}-\frac{1}{8} m_{(2,1 ; 1)}+\frac{3}{4} m_{(1,0 ; 1,1,1)} .
\end{aligned}
$$

By contrast, the limits $t \rightarrow 0, \infty$ depicted in Fig. 3 respectively lead to

$$
\begin{gathered}
s_{(1,0 ; 3)}=m_{(1,0 ; 3)}+m_{(2,0 ; 2)}+m_{(1,0 ; 2,1)}+m_{(2,0 ; 1,1)}+m_{(1,0 ; 1,1,1)}, \\
\bar{s}_{(1,0 ; 3)}=m_{(1,0 ; 3)}+m_{(1,0 ; 2,1)}+m_{(1,0 ; 1,1,1)} .
\end{gathered}
$$

From the consideration of all the examples we could construct (cf. Table 1), we formulate the following conjecture.

Conjecture 6. The expansion coefficients $K_{\Lambda \Omega}$ and $\bar{K}_{\Lambda \Omega}$ defined by

$$
s_{\Lambda}=\sum_{\Omega \leq \Lambda} \bar{K}_{\Lambda \Omega} m_{\Omega} \quad \text { and } \quad \bar{s}_{\Lambda}=\sum_{\Omega \leq \Lambda} K_{\Lambda \Omega} m_{\Omega}
$$

are non-negative integers.

It is interesting and somewhat surprising to see that in superspace, the Schur polynomials with positive coefficients cannot be reached from the Jack side but solely from the Hall-Littlewood ones. Even more remarkably, it appears that the positivity of the change of basis matrix between the Schur and Hall-Littlewood polynomials generalizes to superspace.

Conjecture 7. The expansion coefficients $K_{\Lambda \Omega}(t)$ and $\bar{K}_{\Lambda \Omega}(t)$ defined by

$$
s_{\Lambda}=\sum_{\Omega \leq \Lambda} \bar{K}_{\Lambda \Omega}(t) P_{\Omega}(t) \quad \text { and } \quad \bar{s}_{\Lambda}=\sum_{\Omega \leq \Lambda} K_{\Lambda \Omega}(t) \bar{P}_{\Omega}(1 / t)
$$

are polynomials in $t$ with nonnegative integer coefficients. Furthermore, when $t=1$ they satisfy $\bar{K}_{\Lambda \Omega}(1)=\bar{K}_{\Lambda \Omega}$ and $K_{\Lambda \Omega}(1)=K_{\Lambda \Omega}$.

We end this section by presenting an outstanding conjecture that both supports the correctness of our construction of the Macdonald superpolynomials and the related definitions of the Schur superpolynomials: the superspace version of the Macdonald positivity conjecture (see [14] or [15], eq VI (8.18?), and [12] for its proof). Introduce first the endomorphism $\varphi$ of $\mathbb{Q}(q, t)\left[p_{1}, p_{2}, p_{3}, \ldots ; \tilde{p}_{0}, \tilde{p}_{1}, \tilde{p}_{2}, \ldots\right]$, defined by its action on the power-sums a: 5

$$
\varphi\left(p_{n}\right)=\left(1-t^{n}\right) p_{n} \quad \text { and } \quad \varphi\left(\tilde{p}_{n}\right)=\tilde{p}_{n} .
$$

We then introduce a deformation of the Schur superpolynomials $s_{\Lambda}(x, \theta)$ as

$$
S_{\Lambda}(x, \theta ; t)=\varphi\left(s_{\Lambda}(x, \theta)\right) .
$$

Conjecture 8. The coefficients $K_{\Omega \Lambda}(q, t)$ in the expansion of the integral form of the Macdonald superpolynomials (see Conjecture 3 )

$$
J_{\Lambda}(x, \theta ; q, t)=\sum_{\Omega} K_{\Omega \Lambda}(q, t) S_{\Omega}(x, \theta ; t)
$$

are polynomials in $q$ and $t$ with nonnegative integer coefficients (tables of coefficients $K_{\Omega \Lambda}(q, t)$ can be found at the end of the article).

\footnotetext{
${ }^{5}$ This endomorphism corresponds in the non-fermionic case to the operation that sends $f[X]$ to $f[X(1-t)]$ in $\lambda$-ring notation. This operation is called "plethysm" by some authors (see for instance Section 3.8 of [1]).
} 
Although the Macdonald positivity conjecture in superspace is formulated in terms of the family of Schur superpolynomials $s_{\Omega}$, our computer calculations revealed that the generalized $q, t$-Kostka coefficients $K_{\Omega \Lambda}(q, t)$ are related to the second family $\bar{s}_{\Lambda}$ in a very elegant way.

Conjecture 9. We have $K_{\Omega \Lambda}=K_{\Omega \Lambda}(0,1)$ and $K_{\Omega \Lambda}(t)=K_{\Omega \Lambda}(0, t)$.

\section{Conclusion And OUtLOOK}

Since the discovery of the Jack superpolynomials [3, a natural question has been to determine their Macdonald counterpart. The obvious starting point was to look for the supersymmetric version of the trigonometric Ruijsenaars-Schneider model and define the Macdonald superpolynomials as the common eigenfunctions of all the conservation laws. However, no such candidate Hamiltonian has been found so far: this is still an open problem. We stress that to demonstrate the existence of the Macdonald superpolynomials it is almost imperative to determine the operators that have them as eigenfunctions.

Under these circumstances, it is natural to proceed by brute force and investigate the existence of Macdonald polynomials in superspace constructed along a precise procedure (triangularity and orthogonality). This type of construction leads to over-determined systems of equations that only in a few remarkable cases have a solution. The experimental confirmation that the over-determined systems of equations we obtain have a solution (cf. Table 1) gives strong evidence that we have defined the right Macdonald polynomials in superspace. We got further confirmation of the correctness of the characterization (4.11) by examining in Section 4.3 three other combinatorial properties, which happen to have the expected form generalizing what was obtained in the Jack superpolynomial case.

In addition, we have identified (to be published elsewhere) the vanishing conditions generalizing [10] (see [9] in the Jack's case and [7] for the superspace version). The essential modification here is that the admissibility condition $\lambda_{i}-\lambda_{k+i} \geq r$ of [10] is changed to $\Lambda_{i}^{\circledast}-\Lambda_{i+k}^{*} \geq r 6$

The discovery of combinatorially richer versions of the Schur functions in superspace also open many avenues. For instance, new tableaux combinatorics should explain the monomial positivity of $s_{\Lambda}$ and $\bar{s}_{\Lambda}$, and a connection with representation theory could hopefully be unraveled.

But what appears to be the strongest validation of the present construction is clearly the superspace version of the Macdonald positivity conjecture. In a forthcoming publication, we will present other conjectures related to the $q, t$-Kostka coefficients in superspace, such as symmetry properties that generalize VI (8.14) and VI (8.15) of [15]. One of those conjectures states explicitly how the $q, t$-Kostka coefficients provide a non-trivial refinement of the $q, t$-Kostka coefficients ( $q, t$-Kostka coefficients appear to be positive sums of $q, t$-Kostka coefficients in superspace). We can thus hope that the $q, t$-Kostka coefficients in superspace will help shed some light on the famously difficult combinatorics of the $q, t$-Kostka coefficients.

Acknowledgments. We thank the referee for his constructive comments. This work was supported by NSERC, FQRNT, FONDECYT (Fondo Nacional de Desarrollo Científico y Tecnológico de Chile) grants \#1090016 and \#1090034, and by CONICYT (Comisión Nacional de Investigación Científica y Tecnológica de Chile) via the Redes De Colaboración RED4 and the Programa de Investigación Asociativa ACT56.

\footnotetext{
${ }^{6}$ Quite interestingly, such admissible superpartitions for $r=2$ describe the basis of states in the superconformal minimal models $\mathcal{S M}(2,4 k+4)$ (see [17, 11, Appendix] and the introduction of [7] for more details).
} 


\section{REFERENCES}

[1] F. Bergeron, Algebraic Combinatorics and Coinvariant Spaces, CMS Treatise in Mathematics, A.K. Peters Publishers, 2009.

[2] P. Desrosiers, L. Lapointe and P. Mathieu, Supersymmetric Calogero-Moser-Sutherland models and Jack superpolynomials, Nucl. Phys. B606 (2001), 547-582.

[3] P. Desrosiers, L. Lapointe and P. Mathieu, Jack polynomials in superspace, Commun. Math. Phys. 242 (2003), 331-360.

[4] P. Desrosiers, L. Lapointe and P. Mathieu, Classical symmetric functions in superspace, J. Alg. Comb. 24 (2006), 209-238.

[5] P. Desrosiers, L. Lapointe and P. Mathieu, Orthogonality of Jack polynomials in superspace, Adv. Math. 212 (2007), 361-388.

[6] P. Desrosiers, L. Lapointe and P. Mathieu, Evaluation and normalization of Jack polynomials in superspace, Int. Math Res. Notices doi:10.1093/imrn/rnr235, 61 pages, arXiv:1104.3260.

[7] P. Desrosiers, L. Lapointe and P. Mathieu, Jack superpolynomials with negative fractional parameter: clustering properties and super-Virasoro ideals, arXiv:1109.2832 1.

[8] J. F. van Diejen, On the diagonalization of difference Calogero-Sutherland systems, in Symmetries and integrability of difference equations, CRM Proceedings and Lecture Notes, vol. 9, ed. by D. Levi et al, AMS (1996), 79-89.

[9] B. Feigin, M. Jimbo, T. Miwa and E. Mukhin, A differential ideal of symmetric polynomials spanned by Jack polynomials at $\beta=-(r-1) /(k+1)$, Int. Math. Res. Not. (2002) 23, 1223-1237.

[10] B. Feigin, M. Jimbo, T. Miwa and E. Mukhin, Symmetric polynomials vanishing on the shifted diagonals and Macdonald polynomials, Int. Math. Res. Notices (2003) 18, 1015-1034.

[11] J.-F. Fortin, P. Jacob, P. Mathieu, $\mathcal{S} \mathcal{M}(2,4 \kappa)$ fermionic characters and restricted jagged partitions, J. Phys. A38 (2005) 1699-171.

[12] M. Haiman, Hilbert schemes, polygraphs, and the Macdonald positivity conjecture, J. Amer. Math. Soc. 14 (2001) 941??006.

[13] L. Lapointe, Y. Le Borgne and P. Nadeau A normalization formula for the Jack polynomials in superspace and an identity on partitions, Electronic J. Comb. 16 (2009) Article \#R70.

[14] I. G. Macdonald, Some conjectures for root systems, SIAM J. Math. Anal. 13 (1982) $988 ? ? 007$.

[15] I. G. Macdonald, Symmetric functions and Hall polynomials, 2nd ed., Clarendon Press, 1995.

[16] I. G. Macdonald, Symmetric functions and orthogonal polynomials, University Lecture Series, vol 12, AMS, 1998.

[17] E. Melzer, Supersymmetric analogs of the Gordon-Andrews identities, and related TBA systems, hep-th/9412154

[18] S. N. Ruijsenaars, Systems of Calogero-Moser type, in Particles and fields, CRM series in Mathematical physics, ed. by G. Semenoff and L. Vinet, Springer (1999), 251-352.

[19] S. N. Ruijsenaars and H. Schneider A new class of integrable systems and its relation to solitons, Ann. Phys. 170 (1986), 370-405; S. N. Ruijsenaars, Complete integrability of relativistic Calogero-Moser systems and elliptic function identities, Commun. Math. Phys. 110 (1987), 191-213.

[20] B. S. Shastry and B. Sutherland, Superlax pairs and infinite symmetries in the $1 / r^{2}$ system, Phys. Rev. Lett. 70(1993), 4029-4033; L. Brink, T. H. Hansson, S. Konstein and M. A. Vasiliev, The Calogero model: Anyonic representation, fermionic extension and supersymmetry, Nucl. Phys. B401 (1993) 591.

[21] J. R. Stembridge, A characterization of supersymmetric polynomials, J. Alg. 95 (1985) 439. 
DÉpartement de Physique, de GÉnie physique et D’optique, Université Laval, Québec, Canada, G1V 0A6.

E-mail address: olivier.b-fournier.1@ulaval.ca

Instituto de Matemática y Física, Universidad de Talca, Casilla 747, Talca, Chile.

E-mail address: patrick@inst-mat.utalca.cl

Instituto de Matemática y Física, Universidad de Talca, Casilla 747, Talca, Chile.

E-mail address: lapointe@inst-mat.utalca.cl

Département de Physique, De GÉnie physique et D’optique, Université Laval, Québec, Canada, G1V 0A6.

E-mail address: pmathieu@phy.ulaval.ca 
TABLE 2. Sample of small degree Macdonald polynomials in superspace expanded in the monomial basis (excluding those containing a single monomial term).

\begin{tabular}{|c|c|}
\hline $\operatorname{deg}(\Lambda)$ & $P_{\Lambda}$ \\
\hline$(1 \mid 1)$ & $P_{(1 ;)}=m_{(1 ;)}+\frac{q(1-t)}{1-q t} m_{(0 ; 1)}$ \\
\hline$(2 \mid 1)$ & $\begin{array}{l}P_{(1 ; 1)}=m_{(1 ; 1)}+\frac{q\left(1-t^{2}\right)}{1-q t^{2}} m_{(0 ; 1,1)} \\
P_{(0 ; 2)}=m_{(0 ; 2)}+\frac{1-t}{1-t} m_{(1 ; 1)}+\frac{(1+q)(1-t)}{1-q t} m_{(0 ; 1,1)} \\
P_{(2 ;)}=m_{(2 ;)}+\frac{q^{2}(1-t)}{1-q^{2} t} m_{(0 ; 2)}+\frac{q(1+q)(1-t)}{1-q^{2} t} m_{(1 ; 1)}+\frac{q^{2}(1+q)(1-t)^{2}}{(1-q t)\left(1-q^{2} t\right)} m_{(0 ; 1,1)}\end{array}$ \\
\hline$(2 \mid 2)$ & $P_{(2,0 ;)}=m_{(2,0 ;)}+\frac{q(1-t)}{1-q t} m_{(1,0 ; 1)}$ \\
\hline$(3 \mid 2)$ & $\begin{aligned} P_{(1,0 ; 2)}= & m_{(1,0 ; 2)}+\frac{(1+q t)(1-t)}{1-q t^{2}} m_{(1,0 ; 1,1)} \\
P_{(2,0 ; 1)}= & m_{(2,0 ; 1)}+\frac{q(1-t)}{1-q t} m_{(1,0 ; 2)}+\frac{2 q(1-t)}{1-q t} m_{(1,0 ; 1,1)} \\
P_{(2,1 ;)}= & m_{(2,1 ;)}+\frac{q(1-t)}{1-q t} m_{(2,0 ; 1)}-\frac{q^{2} t(1-q)(1-t)}{(1+q t)(1-q t)^{2}} m_{(1,0 ; 2)}+\frac{q^{2}(1-t)^{2}}{(1-q t)^{2}} m_{(1,0 ; 1,1)} \\
P_{(3,0 ;)}= & m_{(3,0 ;)}+\frac{q(1-t)}{1-q^{2} t} m_{(2,1 ; 0)}+\frac{q(1+q)(1-t)}{1-q^{2} t} m_{(2,0 ; 1)}+\frac{q^{2}(1-t)}{1-q^{2} t} m_{(1,0 ; 2)} \\
& \quad+\frac{q^{2}(1+q)(1-)^{2}}{(1-q t)\left(1-q^{2} t\right)} m_{(1,0 ; 1,1)}\end{aligned}$ \\
\hline$(4 \mid 1)$ & 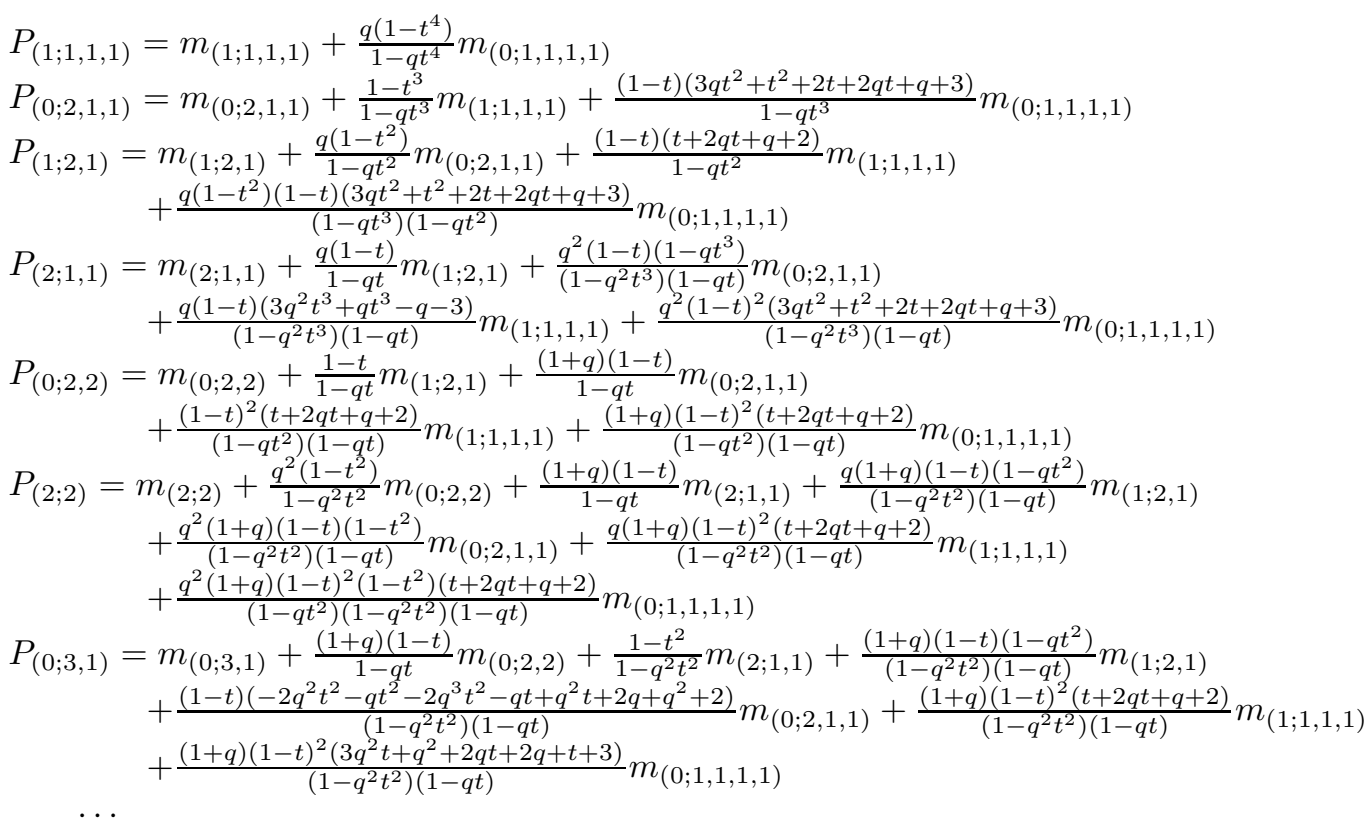 \\
\hline
\end{tabular}


TABLE 3. $K_{\Omega \Lambda}(q, t)$ for degree $(1 \mid 1)$.

\begin{tabular}{c|c|c|} 
& $(1 ;)$ & $(0 ; 1)$ \\
\hline$(1 ;)$ & 1 & $q$ \\
\hline$(0 ; 1)$ & $t$ & 1 \\
\hline
\end{tabular}

TABLE $4 . \quad K_{\Omega \Lambda}(q, t)$ for degree $(2 \mid 1)$.

\begin{tabular}{c|c|c|c|c|} 
& $(2 ;)$ & $(0 ; 2)$ & $(1 ; 1)$ & $(0 ; 1,1)$ \\
\hline$(2 ;)$ & 1 & $q^{2}$ & $q$ & $q^{3}$ \\
\hline$(0 ; 2)$ & $t$ & 1 & $q t$ & $q$ \\
\hline$(1 ; 1)$ & $t$ & $q t$ & 1 & $q$ \\
\hline$(0 ; 1,1)$ & $t^{3}$ & $t$ & $t^{2}$ & 1 \\
\hline
\end{tabular}

TABLE $5 . K_{\Omega \Lambda}(q, t)$ for degree $(2 \mid 2)$.

\begin{tabular}{c|c|c|} 
& $(2,0 ;)$ & $(1,0 ; 1)$ \\
\hline$(2,0 ;)$ & 1 & $q$ \\
\hline$(1,0 ; 1)$ & $t$ & 1 \\
\hline
\end{tabular}

TABLE $6 . \quad K_{\Omega \Lambda}(q, t)$ for degree $(3 \mid 1)$.

\begin{tabular}{c|c|c|c|c|c|c|c|} 
& $(3 ;)$ & $(0 ; 3)$ & $(2 ; 1)$ & $(1 ; 2)$ & $(0 ; 2,1)$ & $(1 ; 1,1)$ & $(0 ; 1,1,1)$ \\
\hline$(3 ;)$ & 1 & $q^{3}$ & $q+q^{2}$ & $q^{2}+q^{4}$ & $q^{4}+q^{5}$ & $q^{3}$ & $q^{6}$ \\
\hline$(0 ; 3)$ & $t$ & 1 & $q t+q^{2} t$ & $q+q^{2} t$ & $q+q^{2}$ & $q^{3} t$ & $q^{3}$ \\
\hline$(2 ; 1)$ & $t$ & $q^{2} t$ & $1+q t$ & $q+q^{2} t$ & $q^{2}+q^{3} t$ & $q$ & $q^{3}$ \\
\hline$(1 ; 2)$ & $t^{2}$ & $q t$ & $t+q t^{2}$ & $1+q^{2} t^{2}$ & $q+q^{2} t$ & $q t$ & $q^{2}$ \\
\hline$(0 ; 2,1)$ & $t^{3}$ & $t$ & $t^{2}+q t^{3}$ & $t+q t^{2}$ & $1+q t$ & $q t^{2}$ & $q$ \\
\hline$(1 ; 1,1)$ & $t^{3}$ & $q t^{3}$ & $t+t^{2}$ & $t+q t^{2}$ & $q t+q t^{2}$ & 1 & $q$ \\
\hline$(0 ; 1,1,1)$ & $t^{6}$ & $t^{3}$ & $t^{4}+t^{5}$ & $t^{2}+t^{4}$ & $t+t^{2}$ & $t^{3}$ & 1 \\
\hline
\end{tabular}

TABLE $7 . \quad K_{\Omega \Lambda}(q, t)$ for degree $(3 \mid 2)$.

\begin{tabular}{c|c|c|c|c|c|} 
& $(3,0 ;)$ & $(2,1 ;)$ & $(2,0 ; 1)$ & $(1,0 ; 2)$ & $(1,0 ; 1,1)$ \\
\hline$(3,0 ;)$ & 1 & $q$ & $q+q^{2}$ & $q^{2}$ & $q^{3}$ \\
\hline$(2,1 ;)$ & $q t$ & 1 & $q+q^{2} t$ & $q^{3} t$ & $q^{2}$ \\
\hline$(2,0 ; 1)$ & $t$ & $t$ & $1+q t$ & $q$ & $q$ \\
\hline$(1,0 ; 2)$ & $t^{2}$ & $q t^{3}$ & $t+q t^{2}$ & 1 & $q t$ \\
\hline$(1,0 ; 1,1)$ & $t^{3}$ & $t^{2}$ & $t+t^{2}$ & $t$ & 1 \\
\hline
\end{tabular}

